\title{
Modelación de los efectos de la compensación de potencia reactiva en sistemas de suministro eléctrico
}

\author{
Modelation of effects on reactive power compensation in secondary power systems
}

Davel Borges Vasconcellos ${ }^{1} \quad$ Ignacio Pérez Abril ${ }^{2} \quad$ Vicente León Martínez $^{3}$

Recibido 3 de junio de 2011, aceptado 30 de julio de 2012

Received: June 3, $2011 \quad$ Accepted: July 30, 2012

\begin{abstract}
RESUMEN
Se presentan los inconvenientes de la potencia reactiva y a partir de allí se realiza la modelación matemática de los efectos económicos de ésta, aplicable a sistemas de suministro eléctrico.

Los efectos tomados en cuenta son: los costos de inversión de los bancos de capacitores, los gastos de amortización de los bancos, los gastos de pérdidas de energía activa de los bancos, los beneficios por la mejora del factor de potencia, incluida la reducción de pérdidas de energía activa en el sistema, así como los beneficios por la liberación de la capacidad de carga, para el caso de empresas donde este aspecto pueda representar un atractivo económico.

Para el análisis de los estados de carga antes y después de la compensación se utilizan algoritmos de flujos de potencia trifásicos, con el empleo de modelos de gráficos de carga ajustados, como aspecto novedoso. Estos efectos, de acuerdo con el sistema de tarifas eléctricas de Cuba, son integrados en una función de análisis económico, en este caso el Valor Actual Neto $(V A N)$, la cual sirve de base para el planteamiento del problema de optimización, que puede derivar en la correcta selección de los dispositivos compensadores.

Palabras clave: Compensación reactiva, modelos de compensación, factor de potencia, sistemas de suministro eléctrico, optimización de potencia reactiva.

ABSTRACT

The inconveniences of the reactive power are presented and from this are carried out the mathematical models of its economic effects applicable to secondary power systems.

The effects taken into account are: the costs of the capacitors banks, the expenses of paying-off to the banks, the expenses of power losses to the banks, the benefits for the improvement of the power factor, included the reduction of power loss in the system, as well as the benefits for the liberation of the load capacity, to the case of companies where the latest aspect can represent an economic attractiveness. For the analysis after and before compensation, are consider load flow algorithm witch load model adjust. According to electrical tariff structures in Cuba, these effects are integrated in a function of economic analysis, for this case the net actual value, which serves as base for the position of the optimization problem, which can derive in the correct selection of the devices compensators.
\end{abstract}

Keywords: Reactive compensation, compensation models, power factor, secondary power systems, reactive power compensations.

\footnotetext{
1 Departamento de Ingeniería Eléctrica. Universidad de Camagüey. Circunvalación Norte Km 51̌․ Camagüey, Cuba. E-mail: davel.borges@ reduc.edu.cu

2 Centro de Estudios Electroenergéticos. Universidad Central Marta Abreu de Las Villas. Carretera de Camajuaní Km 51ำ. Santa Clara, Cuba. E-mail: iperez@uclv.edu.cu

3 Departamento de Ingeniería Eléctrica. Universidad Politécnica de Valencia. Camino de Vera s/n. Valencia, España. E-mail: vleon@die.upv.es
} 


\section{INTRODUCCIÓN}

Los sistemas de suministro eléctrico, también denominados sistemas de distribución secundaria o sistemas de baja tensión, constituyen un subsistema bien definido dentro del sistema electroenergético nacional, caracterizados por operar generalmente como entidades económicas independientes que se relacionan contractualmente con las empresas de transmisión y distribución de energía eléctrica [1].

Otra cualidad importante es el hecho de que es en este subsistema donde se verifican los aspectos relacionados con la calidad de la energía eléctrica servida al cliente.

Todo lo anterior provoca que la compensación de la potencia reactiva en un sistema de suministro eléctrico se realice con el objetivo de optimizar los costos de la energía eléctrica [2], estimulada en lo fundamental por la normativa de comercialización del servicio eléctrico vigente en el país, que establece, generalmente a través de una cláusula del factor de potencia, un sistema de penalización-bonificación en límites establecidos.

El estricto cumplimiento de esta normativa, comprendida en el sistema de tarifas eléctricas, ha restado interés al problema de la selección del tipo de compensación (fija o variable), así como a la ubicación óptima de los dispositivos de compensación en el sistema; y en muchas ocasiones ha reducido el problema únicamente a la correcta selección de los dispositivos a ubicar a nivel global en busca de máximos beneficios económicos, según la normativa de estimulación del factor de potencia.

No obstante, si bien la reducción de pérdidas en la transferencia de energía, la elevación de la tensión en los nodos de compensación del esquema y la liberación de cargas no son motivos suficientes para acometer desde el punto de vista económico la compensación, pueden constituir elementos a tener en cuenta en el proceso de selección y ubicación de los dispositivos, buscando de forma óptima la reducción de los gastos totales de la energía eléctrica, con lo cual se logra una mayor competitividad, a la vez que apunta hacia un mayor impacto en el plano económico, social y ambiental del país.
En este aspecto la bibliografía clásica propone que desde el punto de vista práctico siempre es económico extraer todas las ventajas de la bonificación. [3].

En el presente trabajo se formulan los modelos matemáticos de los efectos económicos de la compensación de potencia reactiva aplicados a los sistemas de suministro eléctrico, basado en la normativa de facturación del servicio eléctrico de la República de Cuba [4], que posteriormente pueden ser empleados en los procedimientos de optimización con vistas a la correcta selección de los dispositivos compensadores.

\section{ANÁLISIS TEÓRICO}

\section{Formulación de la potencia reactiva, según la teoría actual de la potencia}

Es un hecho ya demostrado la existencia de varias componentes de la potencia relacionadas con los diferentes fenómenos energéticos presentes en los sistemas eléctricos [5], que se expresan en:

a) Transferencia de energía útil, es la energía eléctrica que finalmente se transforma en otro tipo de energía (calorífica, mecánica, radiante, entre otras) en las mejores condiciones de eficiencia y calidad en el suministro. Este flujo unidireccional de energía está caracterizado por la componente efectiva (activa, de frecuencia fundamental y secuencia directa) de las tensiones y corrientes y es cuantificado por la potencia efectiva.

b) Fenómeno reactivo de desfase, se establece como flujo sinusoidal de energía eléctrica que no se transforma y que tiene la misma frecuencia y secuencia de fases que la fundamental del generador. Está caracterizado por las componentes de desfase (reactivas, de frecuencia fundamental y secuencia directa) de las tensiones y corrientes, y se cuantifica por la potencia reactiva o de desfase.

c) Fenómeno de asimetría, se manifiesta en los sistemas polifásicos mediante flujos sinusoidales, de frecuencia fundamental y desbalanceados de energía eléctrica que no se transforman. Está caracterizado por componentes de asimetría (de secuencias inversa y homopolar a la frecuencia 
fundamental) de las tensiones y corrientes, y se cuantifica por la potencia de asimetría.

d) Fenómeno de distorsión, producido por la falta de linealidad, que se manifiesta mediante flujos bidireccioneales, no sinusoidales, de energía eléctrica que no se trasforman. Está caracterizado por componentes de distinta frecuencia en las tensiones y corrientes, y se cuantifica por la potencia de deformación armónica.

Cada uno de estos fenómenos puede ser caracterizado por expresiones matemáticas para diferentes tipos de sistemas eléctricos.

Las respectivas potencias pueden ser representadas por sendos vectores en cuadratura de fase, que forman una figura tetradimensional, denominada hipercubo geométrico.

Para este caso, la potencia reactiva, al igual que el resto de las potencias no activas (de asimetría y de deformación armónica), está asociada a fenómenos indeseables de la transferencia, necesarios de compensar para mejorar la eficiencia de los sistemas eléctricos.

Aun cuando en los sistemas de suministro eléctrico la compensación de potencia reactiva se realiza usualmente mediante bancos de capacitores trifásicos balanceados fijos o variables (controlados), el uso de capacitores asimétricos por fase puede resultar en una mejora adicional de la eficiencia, al compensar parcialmente la potencia de asimetría [6].

Como resultado en los sistemas desbalanceados se pueden evitar efectos nocivos tales como: el efecto Ferranti sobre transformadores de distribución o el mal funcionamiento de los bancos controlados por factor de potencia. Es por ello que la mejor variante resulta la implementación de la compensación reactiva en consideración con la potencia de asimetría.

\section{Efectos de la potencia reactiva en los sistemas eléctricos}

Como es conocido, la potencia reactiva en los sistemas de suministro eléctrico trae aparejado un conjunto de efectos indeseables que se pueden resumir en [7]:
1. Aumento de las pérdidas de potencia y energía eléctrica activa en los elementos del sistema: esto se debe a la circulación de una componente de corriente (reactiva) cuyo valor eficaz ocasiona pérdidas en los elementos del sistema por efecto Joule.

2. Incremento del calentamiento de todos los elementos de la red eléctrica: como resultado de esta corriente, se producen calentamientos innecesarios en el sistema.

3. Aumento de las caídas de tensión: las corrientes reactivas que circulan por los elementos del sistema (principalmente líneas y transformadores) incrementan las caídas de tensión y, por ende, disminuyen la tensión en los receptores eléctricos.

4. Limitación de la capacidad de los elementos del sistema eléctrico hasta llegar a los receptores: la capacidad del sistema se ve limitada por la presencia de corrientes reactivas, sin que ello contribuya directamente a la transferencia de energía útil.

5. Sobredimensionamiento de los elementos del sistema eléctrico hasta llegar a los receptores: desde el punto de vista del diseño, los elementos del sistema deben sobredimensionarse para que soporten las potencias y corrientes reactivas.

6. Incremento de la posibilidad de averías y encarecimiento de los costos de inversión y explotación del sistema.

7. Aumento de los costos externos de la generación eléctrica: debido a que la generación en muchos países es mayoritariamente a través de plantas termoeléctricas, cuyos residuos son altamente contaminantes del medio ambiente.

8. Aumento de los costos de la energía eléctrica en las instalaciones: su influencia es a través de:

$>\mathrm{El}$ aumento de los gastos por pérdidas de energía eléctrica: las pérdidas de energía eléctrica en los elementos del sistema, incrementan el consumo de energía activa $y$, por ende, el gasto de ésta. 
El incremento de la penalización en la factura del servicio eléctrico por la aplicación de la cláusula del factor de potencia: este efecto se considera el de mayor influencia económica.

\section{Modelación económica de los efectos de la compensación}

Como se ha planteado, para los sistemas de suministro eléctrico la compensación de potencia reactiva tiene un efecto global monetario, expresado en la normativa de comercialización del servicio eléctrico, por lo que su factibilidad puede representarse a través de una expresión de análisis económico, como puede ser el Valor Actual Neto (VAN).

Cuando la compensación se realiza a través de bancos de capacitores, los efectos a tener en cuenta son:

$>$ Costos de inversión de los bancos de capacitores $(K t)$.

Gastos de amortización de los bancos $(\mathrm{Ca})$.

Gastos de pérdidas de energía activa de los bancos $(C p)$.

$>$ Beneficios por la mejora del factor de potencia, incluida la reducción de pérdidas de energía activa en el sistema (Bfp).

Beneficios por la liberación de la capacidad de carga $(B C)$ para el caso de empresas donde este aspecto pueda representar un atractivo económico.

De forma general el VAN puede expresarse:

$$
V A N=-K_{t}+\sum_{j=1}^{n} \frac{E_{j}-S_{j}}{(1+T D)^{j}}
$$

En este caso:

$E_{j}$ : Entradas en los años $j: E_{j}=B f p+B c$

$S_{j}:$ Salidas en los años $j: S_{j}=C=C p+C a$

$C$ : Gastos anuales de explotación

$T D$ : Tasa de descuento.

$n$ : Años de explotación.

\section{Modelación de la inversión de los bancos de capacitores}

Los costos de inversión de los bancos de capacitores ya sean fijos o controlados se pueden representar de manera más real a partir de considerar los aspectos siguientes:

Los costos reales de las unidades capacitoras, donde se puede demostrar que los costos específicos (pesos/CkVAr) se reducen en la medida que se incrementan las capacidades (CkVAr) de las unidades, contrario a como es usualmente asumido por varios autores a considerar constantes dichos costos. El costo real y específico de las unidades disminuye con el nivel de tensión permisible de estas, dependiendo de la tensión de línea donde serán ubicadas.

Cuando no se tengan los costos reales de las unidades para un determinado nivel de tensión, se puede definir un factor de costo específico de las unidades capacitoras de determinada tensión permisible, respecto a las de otro nivel de tensión estándar de costo conocido.

Los costos variables de accesorios (armarios, dispositivos de mando y protección, etc.), los que a su vez dependen de la capacidad de tales accesorios, que es función de las unidades capacitoras (tamaño físico, capacidad, nivel de tensión, etc.). Estos costos son considerados usualmente como constantes por otros autores. El costo de los accesorios $(K a)$ se puede modelar, por ejemplo, asumiendo un tamaño estándar igual de las unidades capacitoras independientemente de su capacidad, en función de las capacidades de los armarios eléctricos.

Los costos variables de mano de obra especializada para realizar el montaje $(\mathrm{Km})$, que dependen de la complejidad de los trabajos a realizar, el tipo de trabajo, incluso de la distancia de la comercializadora a la cual serán ejecutados dichos trabajos.

Los costos aproximadamente constantes del regulador varmétrico $(K r)$ y sus accesorios (transformadores de medición, dispositivos de protección, etc.) para el caso de los bancos controlados. 
De esta forma, el modelo de costo total de las unidades capacitoras $\left(K_{t}\right)$ para bancos controlados puede ser representado como la suma de todos los costos considerados:

$K_{t}=\sum_{i=1}^{n} F K v_{i} \cdot \sum_{j=1}^{k} x_{\text {máxij }} \cdot k_{j}+\sum_{i=1}^{n}\left\{\begin{array}{l}K a_{1}, \sum_{j=1}^{k} x_{\text {máxij }} \leq x_{d 1} \\ K a_{2}, x_{d 1} \leq \sum_{j=1}^{k} x_{\text {máxij }} \leq x_{d 2}+\ldots \\ \vdots \\ K a_{t}, x_{d t-1} \leq \sum_{j=1}^{k} x_{\text {imáxij }} \leq x_{d t}\end{array}\right.$

$\ldots+K r+K m$

Donde:

$F K v_{i}$ : Factor de costo específico de tensión para el nodo $i$.

$x_{\text {máxij }}$ : Elementos de tipo $j$ a ubicar en el nodo $i$ de la matriz $\left[x_{\text {máx }}\right]$ de cantidad de unidades máximas, de orden $k$ tipos de unidades por $n$ números de nodos considerados, donde serán ubicados los bancos.

$k_{j}$ : Componentes de costo real de las unidades de determinado nivel de tensión del vector columna $[k]$ de costos, de orden $k$ tipos de unidades.

$k a_{1}, k a_{2}, \ldots, k a_{t}$ : Costos de accesorios específicos, en función de la capacidad de los armarios eléctricos.

$x_{d 1}, x_{d 2}, \ldots, x_{d t}$ : Capacidad en cantidad de unidades límite de los armarios.

\section{Modelación de los gastos anuales de explotación}

Los dispositivos de compensación de potencia reactiva traen asociados gastos de explotación. Este constituye uno de los efectos netamente negativo sobre el sistema, debido a que la instalación de los compensadores puede incrementar los gastos por pérdidas de energía activa, durante su funcionamiento, así como los gastos asociados a la amortización de estos.

Cuando se emplean dispositivos estáticos de compensación, las pérdidas específicas de potencia activa se encuentran entre 2,5 y 5 W/CkVAr del dispositivo, lo cual es un valor muy pequeño comparado con los beneficios que ocasiona la reducción de las pérdidas del sistema en general.

Por su parte, para bancos de capacitores estáticos el coeficiente anual de descuento por amortización en Cuba está regulado a un valor de 0,075 (7,5\%).

Si se considera que los dispositivos de compensación son estáticos (bancos de capacitores), sus gastos de mantenimiento son pequeños y se pueden despreciar.

Por lo tanto, los gastos anuales de explotación $(C)$ dependen en lo fundamental de los gastos asociados a las pérdidas de energía activa $(C p)$ y los gastos de amortización $(\mathrm{Ca})$ :

$C=C p+C a=\Delta P a q \cdot K c o m b \cdot \sum_{k=1}^{m} X_{k} \cdot t_{k} \cdot T h_{k}+\Phi \cdot K_{t}$

Donde:

$\triangle P a q$ : Pérdidas de potencia activa específicas por unidad de potencia reactiva de compensación instalada, de acuerdo a las especificaciones del fabricante.

$X_{k}$ : Potencia reactiva de compensación del estado $k$.

$t_{k}$ : Tiempo mensual de estado $k$.

$T h_{k}$ : Tarifa horaria de estado $k$ de facturación.

Kcomb: Factor de ajuste de los precios del combustible empleado para la facturación.

$\Phi$ : Coeficiente anual de descuento por amortización.

\section{Modelación de la mejora del factor de potencia y pérdidas de energía}

El gasto mensual de factor de potencia antes de la compensación $(C f p)$ puede determinarse a partir de la reglamentación vigente, en dependencia del valor del factor de potencia medio pesado $(\cos \varphi m)$ a nivel global determinado, como parte del proceso de facturación del servicio eléctrico.

De esta forma antes de la compensación de potencia reactiva: 


$$
C f p \begin{cases}\text { Ifb }\left(\frac{0,9}{\cos \phi m}-1\right) & \text { si } \quad \cos \phi m<0,9 \\ 0 & \text { si } \quad 0,9 \leq \cos \phi m \leq 0,92 \\ \text { Ifb }\left(\frac{0,92}{\cos \phi m}-1\right) & \text { si } \quad 0,92\langle\cos \phi m \leq 0,96 \\ \text { Ifb }\left(\frac{0,92}{0,96}-1\right)=-0,0416 \text { Ifb } & \text { si } \quad \cos \phi m \geq 0,96\end{cases}
$$

Donde:

Ifb: Importe de facturación normal o base antes de la compensación, sin tener en cuenta las penalizaciones.

El factor de potencia antes de la compensación se determina:

$$
\cos \phi m=\cos \left(\tan ^{-1}\left(\frac{E r}{E a}\right)\right)=\frac{E a}{\sqrt{E a^{2}+E r^{2}}}
$$

Siendo:

Ea y Er: Energía activa y reactiva antes de la compensación, respectivamente.

Los valores de energía activa y reactiva antes de la compensación pueden ser obtenidos a través de los registros de consumo mensual de la empresa.

A partir de la demanda contratada $(P c)$ y la energía activa $(E a)$ consumida puede determinarse el importe de facturación normal mensual (sin tener en cuenta las penalizaciones) (Ifb) a partir de la reglamentación vigente.

Luego de la compensación el gasto por concepto de factor de potencia ( $C f p^{\prime}$ ) se determina de forma similar:

$$
C f p^{\prime} \begin{cases}I f b^{\prime}\left(\frac{0,9}{\cos \phi m^{\prime}}-1\right) & \text { si } \cos \phi^{l} m<0,9 \\ 0 & \text { si } 0,9 \leq \cos \phi^{l} m \leq 0,92 \\ I f b^{\prime}\left(\frac{0,92}{\cos \phi m^{\prime}}-1\right) & \text { si } 0,92<\cos \phi^{l} m \leq 0,96 \\ I f b^{\prime}\left(\frac{0,92}{0,96}-1\right)=-0,0416 \text { Ifb } & \text { si } \cos \phi^{l} m \geq 0,96\end{cases}
$$

Donde:

Ifb': Importe de facturación normal después de la compensación, sin tener en cuenta las penalizaciones.
El factor de potencia después de la compensación se determina:

$$
\cos \phi m^{\prime}=\cos \left(\tan ^{-1}\left(\frac{E r^{\prime}}{E a^{\prime}}\right)\right)=\frac{E a^{\prime}}{\sqrt{E a^{\prime 2}+E r^{\prime 2}}}
$$

Siendo:

$E a^{\prime}$ y $E r$ ': Energía activa y reactiva luego de la compensación, respectivamente.

Por su parte, para determinar los valores de energía activa y reactiva luego de la compensación en sistemas desbalanceados es necesario el empleo de algoritmos de flujo de potencia trifásicos, con la presencia de los dispositivos compensadores.

Los beneficios por la mejora del factor de potencia (Bfp) se determinan restando los gastos por este concepto antes y después de la compensación:

$$
B f p=C f p-C f p^{\prime}
$$

\section{Algoritmo de flujo de potencia trifásico}

Los flujos de potencia se aplican a los sistemas eléctricos con la finalidad de determinar las tensiones en todos los nodos del mismo con respecto a un nodo de referencia (real o ficticio).

Una vez conocidas las tensiones, pueden determinarse las transferencias de corrientes y potencias por todas las ramas y elementos del sistema y de esta forma establecer el estado de carga.

El análisis de redes eléctricas en estado estable se realiza usualmente mediante programas de flujo de potencia monofásico. Estos programas consideran la red totalmente simétrica y la carga igualmente balanceada entre las tres fases, lo que no existe en ninguna de las redes reales. Por otra parte, al utilizar esta simplificación, se pierde la posibilidad de estudiar la posible asimetría de las tensiones y su influencia en la calidad de la energía eléctrica suministrada a los receptores.

Tanto en las redes eléctricas de instalaciones industriales, como en las de instalaciones comerciales o de servicio, aparecen situaciones que ameritan el uso de programas de flujo de potencia trifásico, como son: 
Empleo de cargas monofásicas conectadas entre fases o entre fase y neutro.

$\checkmark$ Empleo de bancos de transformadores asimétricos, compuestos por transformadores de capacidad diferente e incluso bancos abiertos y que utilizan conexiones asimétricas (delta con tap central aterrado, etc.).

$\checkmark \quad$ Empleo de alimentadores de cuatro, tres y dos hilos, trifásicos y monofásicos.

En este análisis se sugiere un algoritmo para la resolución del flujo de potencia trifásico, que se basa en la siguiente secuencia de cálculos [8]:

$$
\begin{aligned}
& V_{2}^{0}=-Y_{22}^{-1} Y_{21} V_{1} \\
& k=0 \\
& \text { Repetir } \\
& \quad k \quad=k+1 \\
& \quad I_{2}^{k}=f\left(S_{2}, V_{2}^{k-1}\right) \\
& \quad V_{2}^{k}=Y_{22}^{-1} I_{2}^{k}+V_{2}^{0} \\
& \text { Hasta que } V_{2}^{k} \approx V_{2}^{k-1} \\
& I_{1}=Y_{11} V_{1}+Y_{12} V_{2}^{k}
\end{aligned}
$$

Siendo:

$V_{l}$ y $V_{2}$ : Vectores de tensiones en los nodos conocidos y de carga (desconocidos) respectivamente.

$I_{1}$ e $I_{2}$ : Vectores de corriente en los nodos conocidos y de carga (desconocidos) respectivamente.

$\mathrm{S}_{2}$ : Vector potencia aparente de carga.

El supraíndice está relacionado con la iteración $k$.

\section{$Y$ : Matriz admitancia del sistema.}

Como se puede apreciar, este acercamiento sigue el método de solución por matriz impedancia que tiene una probada robustez y convergencia y combina estas buenas cualidades con una sencillez que facilita extraordinariamente su programación.

La principal desventaja de este método, las necesidades de memoria para almacenar la matriz impedancia $\left(\mathrm{Y}_{22}{ }^{-1}\right)$, no es importante para el orden de los sistemas medianos y pequeños que se emplean en instalaciones industriales o de servicio.

\section{Modelación de la liberación de capacidad de carga}

La liberación de la capacidad de carga como resultado de la compensación de reactivo puede representar atractivos económicos en aquellas empresas donde la reducción de las pérdidas de energía eléctrica activa por ese concepto pueda ser comercializada a través de algún proveedor eléctrico (empresas de generación de energía o empresas con cogeneración).

Para estos casos, considerando las pérdidas de energía de las unidades capacitoras $(\Delta E x)$ y la reducción de pérdidas de energía activa como resultado de la compensación $(\Delta \Delta E a)$, se puede llegar a establecer la energía disponible $\left(E a_{d i s p}\right)$ :

$$
E a_{\text {disp }}=\Delta \Delta E a-\Delta E x
$$

Así como el costo asociado a su comercialización $\left(\mathrm{Ce} a_{d i s}\right)$ :

$$
C e a_{d i s p}=E a_{d i s p} \cdot \text { Ceea }
$$

Donde:

Ceea: Costo específico de la energía a comercializar según la tarifa acordada.

\section{Modelación del comportamiento mediante gráficos de cargas}

Los modelos tradicionales de los gráficos de cargas eléctricas se basan en utilizar un patrón de comportamiento expresado en la variabilidad de $\mathrm{m}$ estados de cargas (de potencia, activa, reactiva, corriente, etc.) que se obtiene como resultado de mediciones eléctricas con analizadores de redes en el mejor de los casos, durante un ciclo productivo o de servicios característico de la instalación, por lo general en 24 horas, con intervalos de medición constantes, mayores o iguales a una hora, generalmente. En ninguno de los casos se prevé un ajuste de estos gráficos de comportamiento en términos de la energía real consumida durante el intervalo de tiempo de medición, al menos a nivel global. 
Sin embargo, como es sabido los gráficos de carga varían durante cada ciclo productivo o de servicios, dependiendo de factores tecnológicos como el tipo y capacidad de producción o servicio, factores ambientales como la temperatura ambiente y la humedad relativa e incluso factores humanos como los hábitos de trabajo, la disciplina laboral o tecnológica, entre otros.

Esto hace que en la mayoría de los casos los gráficos de carga varíen con el día de la semana y la época del año, lejos de la consideración de un posible comportamiento patrón.

En el caso del sector consumidor terciario (comercial), esta variabilidad aleatoria de los gráficos de carga se hace más notable que en el sector industrial. A manera de ejemplo, en los hoteles del sector turístico, aun cuando el ciclo de servicios se considera 24 horas, el comportamiento de las cargas puede variar a intervalos pequeños, no siendo el mismo durante todos los días de la semana y el mes, dependiendo de factores como el nivel ocupacional, la temperatura de la época del año y el segmento turístico.

De lo anterior se concluye que los modelos de gráficos de cargas tradicionales no son capaces de representar con la exactitud deseada la variabilidad de las cargas.

Un modelo más exacto partiría de contar con los gráficos de carga de todo el año, pero esto sería poco práctico, ocasionaría demoras innecesarias al algoritmo posterior de compensación y, aun así, no existiría la garantía de un comportamiento futuro similar.

El modelo de cargas propuesto por los autores se diseñó teniendo en cuenta las consideraciones siguientes:

Posibilidad real de considerar intervalos de medición necesarios e incluso variables de acuerdo a la variabilidad observada en el gráfico de carga, teniendo en cuenta que a menores intervalos de medición, mayor es la demora de corrida del algoritmo futuro de compensación.

> Utilización de más de un gráfico de carga en los nodos durante el año de medición, establecidos por temporadas de comportamiento regular.
Ajuste de los valores de los gráficos de carga mensuales de cada temporada, a partir de los consumos reales de energía activa y reactiva medidos con metros contadores internos o externos (de facturación).

El modelo de cargas se obtiene a partir de la metodología siguiente:

1. Estudio preliminar del comportamiento de las cargas (potencia activa y reactiva) en el tiempo para establecer los intervalos de medición de cada estado de carga y las temporadas de comportamiento regular de la carga en el año.

2. Con el empleo de analizadores de redes ajustados al intervalo de medición necesario, obtener el comportamiento de la carga regular (potencia activa y reactiva) de cada temporada para cada nodo del sistema.

3. Ajustar los gráficos de carga de potencia activa y reactiva, según el consumo de energía activa y reactiva real medido con instrumentos. Esto se logra a partir del procedimiento siguiente:

Sean $P_{i k}$ y $Q_{i k}$ los valores de potencia activa y reactiva respectivamente del nodo $i$ (de $n$ nodos) y el estado $k$ (de $m$ estados) de una duración de intervalo $T_{k}$ para una temporada de comportamiento de carga regular.

Los nuevos estados $P_{i k m e s}$ y $Q_{i k m e s}$ ajustados para la energía activa $\left(E a_{m e s}\right)$ y reactiva $\left(E r_{m e s}\right)$ conocidos respectivamente durante los meses de facturación se obtienen:

$$
\begin{gathered}
P_{i, j, \text { mes }}=\frac{P_{i k} \cdot E a_{\text {mes }}}{\text { Díasmes } \cdot \sum_{i=1}^{n} \sum_{k=1}^{m} P_{i k} \cdot T_{k}} \\
Q_{i, j, \text { mes }}=\frac{Q_{i k} \cdot E r_{\text {mes }}}{\text { Díasmes } \cdot \sum_{i=1}^{n} \sum_{k=1}^{m} Q_{i k} \cdot T_{k}}
\end{gathered}
$$

Siendo:

Díasmes: Cantidad de días del mes.

Los valores de $P_{i k}, Q_{i k}$ y $T_{k}$ pueden cambiar dependiendo de las temporadas regulares de comportamiento de carga seleccionadas previamente. 


\section{El problema de la compensación de potencia reactiva}

Sobre la base de la modelación de los efectos anteriores se puede formular el problema de la compensación de potencia reactiva como un problema de optimización, en el cual los valores de las variables independientes, resultado del proceso de optimización, serán tomados en cuenta para la correcta selección de los equipos compensadores.

En este caso se recomienda asumir como restricciones del modelo los límites de tensión, así como otros aspectos de calidad de la energía relacionados con el problema de compensación y regulados según las normas [9].

También será posible el empleo de restricciones relacionadas con la limitación del espacio de búsqueda del algoritmo, con vistas a asegurar una convergencia en el menor tiempo posible.

$$
\max \{V A N\}
$$

Sujeto a: $\quad \max [U] \leq U_{\max }$

$$
\min [U] \geq U_{\min }
$$

Donde:

$[U]$ : Matriz de tensiones efectivas, formada por los elementos $U_{i j}$, donde $i$ representa los estados de carga y $j$ los nodos del sistema.

Es necesario aclarar que el modelo presentado es válido para sistemas con baja penetración armónica, por lo cual no han sido tomados en consideración otros efectos relacionados con las distorsiones que pueden modificar el modelo y los futuros resultados.

\section{CONCLUSIONES}

A partir de considerar la compensación de la potencia reactiva en sistemas de suministro eléctrico como un problema económico para las empresas, es posible modelar cada uno de sus efectos e integrarlos en una función de evaluación económica, por ejemplo el Valor Actual Neto $(V A N)$.

Esta formulación es indispensable para la selección adecuada de los dispositivos compensadores, posterior al necesario proceso de optimización, restringido en lo fundamental por el cumplimiento de las normativas de calidad de la tensión aplicable a estos sistemas.

Debido al grado de complejidad y el carácter discreto de las variables, para la solución de este problema se recomienda el uso de algoritmos evolutivos.

\section{AGRADECIMIENTOS}

Los autores agradecen a los profesores: Dr. Leonardo Casas Fernández de la Universidad Central de las Villas, Cuba, y Dr. José Giner García, de la Universidad de Valencia, España, por su ayuda en los basamentos del trabajo.

\section{REFERENCIAS}

[1] I. Pérez Abril and J. González Quintero. "VAR Compensation by Sequential Quadratic Programming". IEEE Transactions on Power Systems. Vol. 18, Issue 1, pp. 36-41. February, 2003.

[2] A. Faheem Zobaa. "Cost-Effective Applications of Power Factor Correction for Nonlinear Loads". IEEE Transactions on Power Delivery. Vol. 20, Issue 1, pp. 359365. January, 2005.

[3] D. Beeman. "Industrial Power Systems Handbook". Ed. McGraw-Hill. New York, USA. 1955.

[4] Resolución Ministerial No 28/2011. "Sistema tarifario para el sector no residencial". Ministerio de Finanzas y Precios. República de Cuba. 19 de enero de 2011.

[5] IEEE Trial-Use Standard Definitions of the Measurement of Electric Power Quantities Under Sinusoidal, Non sinusoidal, Balanced or Unbalanced Conditions. IEEE Std 14592000. /IEEE Power Engineering Society. I Published by the Institute of Electrical and Electronics Engineers, inc., 3 Park Avenue, New York, NY 10016-5997, USA.

[6] V. León Martínez, J. Montañana Romeu, J. Roger Folch and A. Cazorla Navarro. "Why Reactive Compensators do not Improve the Efficiency Correctly in Unbalanced Circuits". XIX IMEKO World Congress Fundamental and Applied Metrology. Lisbon, Portugal. September 6-11, 2009.

[7] V. León Martínez, J. Montañana Romeu, J. Giner García and A. Cazorla Navarro. 
"Ineficiencias de los sistemas eléctricos. Efectos, cuantificación y dispositivos de mejora de la eficiencia”. Ed. Universidad Politécnica de Valencia. España. 2001.

[8] F.M. Gatta, F. Iliceto, S. Lauria and P. Masato. "Analysis of unsymmetrical transmission distribution systems. Application to Insulated Shield Wires Schemes". L'Energia Elettrica. Vol. 79. 2002.

[9] Norma Cubana NC 365: Tensiones normalizadas. Comité Electrotécnico Cubano. Cuba. 2009. 